\title{
Some modular considerations regarding odd perfect numbers - Part II
}

\author{
Jose Arnaldo Bebita Dris ${ }^{1}$ and Immanuel Tobias San Diego ${ }^{2}$ \\ ${ }^{1}$ M. Sc. Graduate, Mathematics Department \\ De La Salle University, Manila, Philippines 1004 \\ e-mail: josearnaldobdris@gmail .com \\ 2 Department of Mathematics and Physical Sciences \\ Trinity University of Asia, Quezon City, Philippines 1102 \\ e-mail: itsandiego@tua.edu.ph
}

Received: 28 February 2020

Revised: 19 July 2020

Accepted: 20 July 2020 \begin{abstract}
$p^{k} m^{2}$ is an odd perfect number with special prime $p$. We prove that
1. $\sigma\left(m^{2}\right) \equiv 1(\bmod 8)$ holds only if $p+k \equiv 2(\bmod 16)$.

2. $\sigma\left(m^{2}\right) \equiv 3(\bmod 8)$ holds only if $p-k \equiv 4(\bmod 16)$.

3. $\sigma\left(m^{2}\right) \equiv 5(\bmod 8)$ holds only if $p+k \equiv 10(\bmod 16)$.

4. $\sigma\left(m^{2}\right) \equiv 7(\bmod 8)$ holds only if $p-k \equiv 4(\bmod 16)$.
\end{abstract}

Abstract: In this article, we consider the various possibilities for $p$ and $k$ modulo 16, and show conditions under which the respective congruence classes for $\sigma\left(m^{2}\right)$ (modulo 8) are attained, if

We express $\operatorname{gcd}\left(m^{2}, \sigma\left(m^{2}\right)\right)$ as a linear combination of $m^{2}$ and $\sigma\left(m^{2}\right)$. We also consider some applications under the assumption that $\sigma\left(m^{2}\right) / p^{k}$ is a square. Lastly, we prove a last-minute conjecture under this hypothesis.

Keywords: Sum of divisors, Sum of aliquot divisors, Deficiency, Odd perfect number, Special prime.

2010 Mathematics Subject Classification: 11A05, 11A25.

\section{Introduction}

Let $\sigma(z)$ denote the sum of the divisors of $z \in \mathbb{N}$, the set of positive integers. Denote the deficiency [13] of $z$ by $D(z)=2 z-\sigma(z)$, and the sum of the aliquot divisors [14] of $z$ by $s(z)=\sigma(z)-z$. Note that we have the identity $D(z)+s(z)=z$. 
If $n$ is odd and $\sigma(n)=2 n$, then $n$ is said to be an odd perfect number [17]. Euler proved that an odd perfect number, if one exists, must have the form $n=p^{k} m^{2}$, where $p$ is the special prime satisfying $p \equiv k \equiv 1(\bmod 4)$ and $\operatorname{gcd}(p, m)=1$.

Chen and Luo [3] gave a characterization of the forms of odd perfect numbers $n=p^{k} m^{2}$ such that $p \equiv k(\bmod 8)$. Starni [16] proved that there is no odd perfect number decomposable into primes all of the type $\equiv 1(\bmod 4)$ if $n=p^{k} m^{2}$ and $p \not \equiv k(\bmod 8)$. Starni used a congruence from Ewell [11] to prove this result.

Note that, in general, since $m^{2}$ is a square, we get

$$
\sigma\left(m^{2}\right) \equiv 1(\bmod 2)
$$

Dris and San Diego [9] provide an alternative proof of the following theorem from Chen and Luo [3]:

Theorem 1.1. If $n=p^{k} m^{2}$ is an odd perfect number with special prime $p$, then $\sigma\left(m^{2}\right) \equiv 1$ $(\bmod 4)$ holds if and only if $p \equiv k(\bmod 8)$.

Chen and Luo [3] actually proved the following (stronger) theorem:

Theorem 1.2. Let $n=p^{k} m^{2}$ be an odd perfect number, with $p$ prime, $\operatorname{gcd}(p, m)=1$, and $p \equiv k \equiv 1(\bmod 4)$. Then

$$
\begin{aligned}
\sigma\left(m^{2}\right) & \equiv 1(\bmod 4) \Longleftrightarrow p \equiv k(\bmod 8), \\
\sigma\left(m^{2}\right) & \equiv 3(\bmod 4) \Longleftrightarrow p \equiv k+4(\bmod 8) .
\end{aligned}
$$

This paper considers the possibilities for $\sigma\left(m^{2}\right)$ modulo 8 under suitable hypotheses for $p$ and $k$ modulo 16.

\section{Preliminaries}

Starting from the fundamental equality

$$
\frac{\sigma\left(m^{2}\right)}{p^{k}}=\frac{2 m^{2}}{\sigma\left(p^{k}\right)}
$$

(which follows from the facts that $\sigma(n)=2 n, \sigma$ is multiplicative, and $\operatorname{gcd}\left(p^{k}, \sigma\left(p^{k}\right)\right)=1$ ) one can derive

$$
\frac{\sigma\left(m^{2}\right)}{p^{k}}=\frac{2 m^{2}}{\sigma\left(p^{k}\right)}=\operatorname{gcd}\left(m^{2}, \sigma\left(m^{2}\right)\right)
$$

so that we ultimately have

$$
\frac{D\left(m^{2}\right)}{s\left(p^{k}\right)}=\frac{2 m^{2}-\sigma\left(m^{2}\right)}{\sigma\left(p^{k}\right)-p^{k}}=\operatorname{gcd}\left(m^{2}, \sigma\left(m^{2}\right)\right)
$$

and

$$
\frac{s\left(m^{2}\right)}{D\left(p^{k}\right) / 2}=\frac{\sigma\left(m^{2}\right)-m^{2}}{p^{k}-\frac{\sigma\left(p^{k}\right)}{2}}=\operatorname{gcd}\left(m^{2}, \sigma\left(m^{2}\right)\right),
$$


whereby we obtain

$$
\frac{D\left(p^{k}\right) D\left(m^{2}\right)}{s\left(p^{k}\right) s\left(m^{2}\right)}=2 .
$$

Note that we also have the following equation

$$
\frac{2 D\left(m^{2}\right) s\left(m^{2}\right)}{D\left(p^{k}\right) s\left(p^{k}\right)}=\left(\operatorname{gcd}\left(m^{2}, \sigma\left(m^{2}\right)\right)\right)^{2} .
$$

Notice that the right-hand side of Equation $(*)$ is odd. (Furthermore, it is congruent to 1 modulo 8.) Lastly, notice that we can easily get

$$
\sigma\left(p^{k}\right) \equiv k+1 \equiv 2(\bmod 4)
$$

(since $p \equiv k \equiv 1(\bmod 4)$ ) so that it remains to consider the possible equivalence classes for $\sigma\left(m^{2}\right)$ modulo 4 .

Chen and Luo proved that $\sigma\left(m^{2}\right) \equiv 1(\bmod 4)$ if and only if $p \equiv k(\bmod 8)$.

This paper considers the following problem: What congruence classes are attained by $\sigma\left(m^{2}\right)$ modulo 8 when $p$ and $k$ are constrained to certain congruence classes modulo 16 ?

\section{Discussion and results}

We know that the answer to the question we posed in the previous section must somehow depend on the equivalence class of $p$ and $k$ modulo 16 , but as we only know that $p \equiv k \equiv 1(\bmod 4)$, and that $p \equiv k(\bmod 8)$ if and only if $\sigma\left(m^{2}\right) \equiv 1(\bmod 4)$, we need to consider the following cases separately and thereby prove the corresponding results.

First, we prove the following lemmas.

Lemma 3.1. Suppose that $n=p^{k} m^{2}$ is an odd perfect number with special prime $p$. Consider the possible congruence classes for $\sigma\left(\mathrm{m}^{2}\right)$ modulo 8.

1. If $\sigma\left(m^{2}\right) \equiv 1(\bmod 8) \operatorname{or} \sigma\left(m^{2}\right) \equiv 5(\bmod 8)$, then $p \equiv k(\bmod 8)$.

2. If $\sigma\left(m^{2}\right) \equiv 3(\bmod 8)$ or $\sigma\left(m^{2}\right) \equiv 7(\bmod 8)$, then $p \not \equiv k(\bmod 8)$.

Proof. This follows directly from Theorem 1.1.

We reproduce the following lemmas from Dris et al. [9], adjusting to account for $p$ and $k$ modulo 16 instead of modulo 8 .

Lemma 3.2. Suppose that $n=p^{k} m^{2}$ is an odd perfect number with special prime $p$.

1. If $p \equiv 1(\bmod 16)$, then $\sigma\left(p^{k}\right) \equiv k+1(\bmod 16)$.

2. If $p \equiv 5(\bmod 16)$, then

$$
\sigma\left(p^{k}\right) \equiv\left\{\begin{array}{lll}
6 & (\bmod 16), \text { if } k \equiv 1 & (\bmod 16) \\
2 & (\bmod 16), \text { if } k \equiv 5 & (\bmod 16) \\
14 & (\bmod 16), \text { if } k \equiv 9 & (\bmod 16) \\
10 & (\bmod 16), \text { if } k \equiv 13 & (\bmod 16)
\end{array}\right.
$$


3. If $p \equiv 9(\bmod 16)$, then

$$
\sigma\left(p^{k}\right) \equiv\left\{\begin{array}{lcc}
10 & (\bmod 16), \text { if } k \equiv 1 & (\bmod 16) \\
14 & (\bmod 16), \text { if } k \equiv 5 & (\bmod 16) \\
2 & (\bmod 16), \text { if } k \equiv 9 & (\bmod 16) \\
6 & (\bmod 16), \text { if } k \equiv 13 & (\bmod 16)
\end{array}\right.
$$

4. If $p \equiv 13(\bmod 16)$, then

$$
\sigma\left(p^{k}\right) \equiv\left\{\begin{array}{lll}
14 & (\bmod 16), \text { if } k \equiv 1 & (\bmod 16) \\
10 & (\bmod 16), \text { if } k \equiv 5 & (\bmod 16) \\
6 & (\bmod 16), \text { if } k \equiv 9 & (\bmod 16) \\
2 & (\bmod 16), \text { if } k \equiv 13 & (\bmod 16)
\end{array}\right.
$$

Proof. Let $n=p^{k} m^{2}$ be an odd perfect number with special prime $p$. It follows that $p \equiv 1$ $(\bmod 4)$.

We consider four cases:

Case 1: $p \equiv 1(\bmod 16)$ We obtain

$$
\sigma\left(p^{k}\right)=\sum_{i=0}^{k} p^{i} \equiv 1+\sum_{i=1}^{k} p^{i} \equiv 1+\sum_{i=1}^{k} 1^{i} \equiv k+1 \quad(\bmod 16),
$$

as desired.

Case 2: $p \equiv 5(\bmod 16)$ We get

$$
\sigma\left(p^{k}\right)=\sum_{i=0}^{k} p^{i} \equiv \sum_{i=0}^{k} 5^{i} \equiv\left\{\begin{array}{lll}
6 & (\bmod 16), \text { if } k \equiv 1 \quad(\bmod 16) \\
2 & (\bmod 16), \text { if } k \equiv 5 \quad(\bmod 16) \\
14 & (\bmod 16), \text { if } k \equiv 9 \quad(\bmod 16) \\
10 & (\bmod 16), \text { if } k \equiv 13 \quad(\bmod 16)
\end{array}\right.
$$

Case 3: $p \equiv 9(\bmod 16)$ We derive

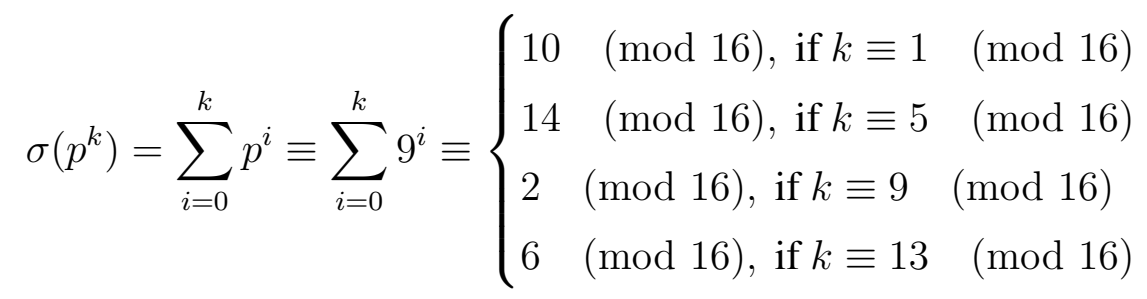

Case 4: $p \equiv 13(\bmod 16)$ We have that

$$
\sigma\left(p^{k}\right)=\sum_{i=0}^{k} p^{i} \equiv \sum_{i=0}^{k} 13^{i} \equiv\left\{\begin{array}{lll}
14 & (\bmod 16), \text { if } k \equiv 1 & (\bmod 16) \\
10 & (\bmod 16), \text { if } k \equiv 5 \quad(\bmod 16) \\
6 & (\bmod 16), \text { if } k \equiv 9 \quad(\bmod 16) \\
2 & (\bmod 16), \text { if } k \equiv 13 \quad(\bmod 16)
\end{array}\right.
$$


The next lemma computes the congruence class for the deficiency of the Euler component $p^{k}$.

Lemma 3.3. Suppose that $n=p^{k} m^{2}$ is an odd perfect number with special prime $p$.

1. Suppose that exactly one of the following conditions hold:

(a) $p \equiv k \equiv 1(\bmod 16)$

(b) $p \equiv 5(\bmod 16), k \equiv 13(\bmod 16)$

(c) $p \equiv k \equiv 9(\bmod 16)$

(d) $p \equiv 13(\bmod 16), k \equiv 5(\bmod 16)$

It follows that $D\left(p^{k}\right) \equiv 0(\bmod 16)$.

2. Suppose that exactly one of the following conditions hold:

(a) $p \equiv 1(\bmod 16), k \equiv 13(\bmod 16)$

(b) $p \equiv 5(\bmod 16), k \equiv 1(\bmod 16)$

(c) $p \equiv 9(\bmod 16), k \equiv 5(\bmod 16)$

(d) $p \equiv 13(\bmod 16), k \equiv 9(\bmod 16)$

It follows that $D\left(p^{k}\right) \equiv 4(\bmod 16)$.

3. Suppose that exactly one of the following conditions hold:

(a) $p \equiv 1(\bmod 16), k \equiv 9(\bmod 16)$

(b) $p \equiv k \equiv 5(\bmod 16)$

(c) $p \equiv 9(\bmod 16), k \equiv 1(\bmod 16)$

(d) $p \equiv k \equiv 13(\bmod 16)$

It follows that $D\left(p^{k}\right) \equiv 8(\bmod 16)$.

4. Suppose that exactly one of the following conditions hold:

(a) $p \equiv 1(\bmod 16), k \equiv 5(\bmod 16)$

(b) $p \equiv 5(\bmod 16), k \equiv 9(\bmod 16)$

(c) $p \equiv 9(\bmod 16), k \equiv 13(\bmod 16)$

(d) $p \equiv 13(\bmod 16), k \equiv 1(\bmod 16)$

It follows that $D\left(p^{k}\right) \equiv 12(\bmod 16)$.

Proof. The proof is trivial and follows directly from Lemma 3.2, using the formula $D\left(p^{k}\right)=$ $2 p^{k}-\sigma\left(p^{k}\right)$.

We now compute the congruence class for the sum of the aliquot divisors of the Euler component $p^{k}$.

Lemma 3.4. Suppose that $n=p^{k} m^{2}$ is an odd perfect number with special prime $p$.

1. Suppose that exactly one of the following conditions hold:

(a) $p \equiv k \equiv 1(\bmod 16)$

(b) $p \equiv 5(\bmod 16), k \equiv 1(\bmod 16)$ 
(c) $p \equiv 9(\bmod 16), k \equiv 1(\bmod 16)$

(d) $p \equiv 13(\bmod 16), k \equiv 1(\bmod 16)$

It follows that $s\left(p^{k}\right) \equiv 1(\bmod 16)$.

2. Suppose that exactly one of the following conditions hold:

(a) $p \equiv 1(\bmod 16), k \equiv 5(\bmod 16)$

(b) $p \equiv 5(\bmod 16), k \equiv 13(\bmod 16)$

(c) $p \equiv 9(\bmod 16), k \equiv 5(\bmod 16)$

(d) $p \equiv 13(\bmod 16), k \equiv 13(\bmod 16)$

It follows that $s\left(p^{k}\right) \equiv 5(\bmod 16)$.

3. Suppose that exactly one of the following conditions hold:

(a) $p \equiv 1(\bmod 16), k \equiv 9(\bmod 16)$

(b) $p \equiv 5(\bmod 16), k \equiv 9(\bmod 16)$

(c) $p \equiv k \equiv 9(\bmod 16)$

(d) $p \equiv 13(\bmod 16), k \equiv 9(\bmod 16)$

It follows that $s\left(p^{k}\right) \equiv 9(\bmod 16)$.

4. Suppose that exactly one of the following conditions hold:

(a) $p \equiv 1(\bmod 16), k \equiv 13(\bmod 16)$

(b) $p \equiv k \equiv 5(\bmod 16)$

(c) $p \equiv 9(\bmod 16), k \equiv 13(\bmod 16)$

(d) $p \equiv 13(\bmod 16), k \equiv 5(\bmod 16)$

It follows that $s\left(p^{k}\right) \equiv 13(\bmod 16)$.

Proof. The proof is trivial and follows directly from Lemma 3.3, using the formula $s\left(p^{k}\right)=p^{k}-D\left(p^{k}\right)$.

Lemma 3.5. Suppose that $n=p^{k} m^{2}$ is an odd perfect number with special prime $p$.

1. If $\sigma\left(m^{2}\right) \equiv 1(\bmod 8)$, then $D\left(m^{2}\right) \equiv 1(\bmod 8)$.

2. If $\sigma\left(m^{2}\right) \equiv 3(\bmod 8)$, then $D\left(m^{2}\right) \equiv 7(\bmod 8)$.

3. If $\sigma\left(m^{2}\right) \equiv 5(\bmod 8)$, then $D\left(m^{2}\right) \equiv 5(\bmod 8)$.

4. If $\sigma\left(m^{2}\right) \equiv 7(\bmod 8)$, then $D\left(m^{2}\right) \equiv 3(\bmod 8)$.

Proof. The proof is trivial and follows directly from the fact that $m^{2} \equiv 1(\bmod 8)$ (since $m$ is odd), using the underlying assumptions and the formula $D\left(m^{2}\right)=2 m^{2}-\sigma\left(m^{2}\right)$.

Lemma 3.6. Suppose that $n=p^{k} m^{2}$ is an odd perfect number with special prime $p$.

1. If $\sigma\left(m^{2}\right) \equiv 1(\bmod 8)$, then $s\left(m^{2}\right) \equiv 0(\bmod 8)$.

2. If $\sigma\left(m^{2}\right) \equiv 3(\bmod 8)$, then $s\left(m^{2}\right) \equiv 2(\bmod 8)$.

3. If $\sigma\left(m^{2}\right) \equiv 5(\bmod 8)$, then $s\left(m^{2}\right) \equiv 4(\bmod 8)$.

4. If $\sigma\left(m^{2}\right) \equiv 7(\bmod 8)$, then $s\left(m^{2}\right) \equiv 6(\bmod 8)$.

Proof. The proof is trivial and follows directly from Lemma 3.5, using the formula $s\left(m^{2}\right)=$ $m^{2}-D\left(m^{2}\right)$. 
We are now ready to prove our main results.

Theorem 3.7. Suppose that $n=p^{k} m^{2}$ is an odd perfect number with special prime $p$ satisfying $\sigma\left(m^{2}\right) \equiv 1(\bmod 8)$. This implies that exactly one of the following conditions hold:

1. $p \equiv k \equiv 1(\bmod 16)$

2. $p \equiv 5(\bmod 16), k \equiv 13(\bmod 16)$

3. $p \equiv k \equiv 9(\bmod 16)$

4. $p \equiv 13(\bmod 16), k \equiv 5(\bmod 16)$

Proof. Let $n=p^{k} m^{2}$ be an odd perfect number with special prime $p$, satisfying $\sigma\left(m^{2}\right) \equiv 1$ $(\bmod 8)$. By Lemma $3.1, p \equiv k(\bmod 8)$ holds.

We now consider each of the resulting possible congruences for $p$ and $k$ modulo 16 :

1. $p \equiv k \equiv 1(\bmod 16)$

2. $p \equiv 1(\bmod 16), k \equiv 9(\bmod 16)$

3. $p \equiv k \equiv 5(\bmod 16)$

4. $p \equiv 5(\bmod 16), k \equiv 13(\bmod 16)$

5. $p \equiv 9(\bmod 16), k \equiv 1(\bmod 16)$

6. $p \equiv k \equiv 9(\bmod 16)$

7. $p \equiv 13(\bmod 16), k \equiv 5(\bmod 16)$

8. $p \equiv k \equiv 13(\bmod 16)$

We shall show that no integer solutions to Equation $(*)$ exist for the second, third, fifth and eighth cases. Notice that the right-hand side of Equation $(*)$ is odd. (Furthermore, it is congruent to 1 modulo 8.)

First, suppose that $p \equiv 1(\bmod 16), k \equiv 9(\bmod 16)$ holds. By Lemma 3.3, $D\left(p^{k}\right) \equiv 8$ $(\bmod 16)$. By Lemma 3.5, $D\left(m^{2}\right) \equiv 1(\bmod 8)$. By Lemma 3.4, $s\left(p^{k}\right) \equiv 9(\bmod 16)$. By Lemma 3.6, $s\left(m^{2}\right) \equiv 0(\bmod 8)$. Thus, from Equation $(*)$ we obtain (symbolically)

$$
2\left(8 a_{1}+1\right)\left(8 b_{1}\right)=\left(8 x_{1}+1\right)\left(16 c_{1}+8\right)\left(16 d_{1}+9\right)
$$

which does not have any integer solutions.

Next, suppose that $p \equiv k \equiv 5(\bmod 16)$ holds. By Lemma $3.3, D\left(p^{k}\right) \equiv 8(\bmod 16)$. By Lemma 3.5, $D\left(m^{2}\right) \equiv 1(\bmod 8)$. By Lemma 3.4, $s\left(p^{k}\right) \equiv 13(\bmod 16)$. By Lemma 3.6, $s\left(m^{2}\right) \equiv 0(\bmod 8)$. Thus, from Equation $(*)$ we obtain (symbolically)

$$
2\left(8 a_{2}+1\right)\left(8 b_{2}\right)=\left(8 x_{2}+1\right)\left(16 c_{2}+8\right)\left(16 d_{2}+13\right)
$$

which does not have any integer solutions.

Now, suppose that $p \equiv 9(\bmod 16), k \equiv 1(\bmod 16)$ holds. By Lemma 3.3, $D\left(p^{k}\right) \equiv 8$ $(\bmod 16)$. By Lemma 3.5, $D\left(m^{2}\right) \equiv 1(\bmod 8)$. By Lemma 3.4, $s\left(p^{k}\right) \equiv 1(\bmod 16)$. By Lemma 3.6, $s\left(m^{2}\right) \equiv 0(\bmod 8)$. Thus, from Equation $(*)$ we obtain (symbolically)

$$
2\left(8 a_{3}+1\right)\left(8 b_{3}\right)=\left(8 x_{3}+1\right)\left(16 c_{3}+8\right)\left(16 d_{3}+1\right)
$$

which does not have any integer solutions. 
Finally, suppose that $p \equiv k \equiv 13(\bmod 16)$ holds. By Lemma 3.3, $D\left(p^{k}\right) \equiv 8(\bmod 16)$. By Lemma 3.5, $D\left(m^{2}\right) \equiv 1(\bmod 8)$. By Lemma 3.4, $s\left(p^{k}\right) \equiv 5(\bmod 16)$. By Lemma 3.6, $s\left(m^{2}\right) \equiv 0(\bmod 8)$. Thus, from Equation $(*)$ we obtain (symbolically)

$$
2\left(8 a_{4}+1\right)\left(8 b_{4}\right)=\left(8 x_{4}+1\right)\left(16 c_{4}+8\right)\left(16 d_{4}+5\right)
$$

which does not have any integer solutions.

It can be double-checked that the other cases yield potential solutions, and do not result to a contradiction.

This concludes the proof.

Theorem 3.8. Suppose that $n=p^{k} m^{2}$ is an odd perfect number with special prime $p$ satisfying $\sigma\left(m^{2}\right) \equiv 3(\bmod 8)$. This implies that exactly one of the following conditions hold:

1. $p \equiv 1(\bmod 16), k \equiv 13(\bmod 16)$

2. $p \equiv 5(\bmod 16), k \equiv 1(\bmod 16)$

3. $p \equiv 9(\bmod 16), k \equiv 5(\bmod 16)$

4. $p \equiv 13(\bmod 16), k \equiv 9(\bmod 16)$

Proof. The proof of this theorem is very similar to that of Theorem 3.7, and is left as an exercise for the interested reader.

Theorem 3.9. Suppose that $n=p^{k} m^{2}$ is an odd perfect number with special prime $p$ satisfying $\sigma\left(m^{2}\right) \equiv 5(\bmod 8)$. This implies that exactly one of the following conditions hold:

1. $p \equiv 1(\bmod 16), k \equiv 9(\bmod 16)$

2. $p \equiv 5(\bmod 16), k \equiv 5(\bmod 16)$

3. $p \equiv 9(\bmod 16), k \equiv 1(\bmod 16)$

4. $p \equiv 13(\bmod 16), k \equiv 13(\bmod 16)$

Proof. The proof of this theorem is very similar to that of Theorem 3.7, and is left as an exercise for the interested reader.

Theorem 3.10. Suppose that $n=p^{k} m^{2}$ is an odd perfect number with special prime $p$ satisfying $\sigma\left(m^{2}\right) \equiv 7(\bmod 8)$. This implies that exactly one of the following conditions hold:

1. $p \equiv 1(\bmod 16), k \equiv 13(\bmod 16)$

2. $p \equiv 5(\bmod 16), k \equiv 1(\bmod 16)$

3. $p \equiv 9(\bmod 16), k \equiv 5(\bmod 16)$

4. $p \equiv 13(\bmod 16), k \equiv 9(\bmod 16)$

Proof. The proof of this theorem is very similar to that of Theorem 3.7, and is left as an exercise for the interested reader.

Remark 3.11. To summarize, Theorem 3.7, Theorem 3.8, Theorem 3.9, and Theorem 3.10 just state collectively that if $n=p^{k} m^{2}$ is an odd perfect number with special prime $p$, then 
1. $\sigma\left(m^{2}\right) \equiv 1(\bmod 8)$ holds only if $p+k \equiv 2(\bmod 16)$.

2. $\sigma\left(m^{2}\right) \equiv 3(\bmod 8)$ holds only if $p-k \equiv 4(\bmod 16)$.

3. $\sigma\left(m^{2}\right) \equiv 5(\bmod 8)$ holds only if $p+k \equiv 10(\bmod 16)$.

4. $\sigma\left(m^{2}\right) \equiv 7(\bmod 8)$ holds only if $p-k \equiv 4(\bmod 16)$.

\section{Applications}

Let $n=p^{k} m^{2}$ be an odd perfect number with special prime $p$, and let $\sigma\left(m^{2}\right) / p^{k}$ be a square. Since $\sigma\left(m^{2}\right) / p^{k}$ is odd, it follows that $\sigma\left(m^{2}\right) / p^{k} \equiv 1(\bmod 4)$. But it is known that $p \equiv k \equiv 1$ $(\bmod 4)$. In particular, we know that $p^{k} \equiv 1(\bmod 4)$. This implies that $\sigma\left(m^{2}\right) \equiv 1(\bmod 4)$, if $\sigma\left(m^{2}\right) / p^{k}$ is a square. By Theorem 1.1 , we infer that $p \equiv k(\bmod 8)$.

Moreover, Broughan, Delbourgo, and Zhou prove in [1, Lemma 8, page 7] that if $\sigma\left(m^{2}\right) / p^{k}$ is a square, then $k=1$ holds.

Thus, under the assumption that $\sigma\left(m^{2}\right) / p^{k}$ is a square, we have

$$
p \equiv k=1 \quad(\bmod 8) .
$$

Remark 4.1. Let $n=p^{k} m^{2}$ be an odd perfect number with special prime $p$.

Note that if

$$
\frac{\sigma\left(m^{2}\right)}{p^{k}}=\frac{m^{2}}{\sigma\left(p^{k}\right) / 2}
$$

is a square, then $k=1$ and $\sigma\left(p^{k}\right) / 2=(p+1) / 2$ is also a square.

The possible values for the special prime satisfying $p<100$ and $p \equiv 1(\bmod 8)$ are 17,41 , 73, 89, and 97. For each of these values:

$$
\begin{aligned}
& \frac{p_{1}+1}{2}=\frac{17+1}{2}=9=3^{2} . \\
& \frac{p_{2}+1}{2}=\frac{41+1}{2}=21, \text { which } \text { is not a square. } \\
& \frac{p_{3}+1}{2}=\frac{73+1}{2}=37, \text { which is not a square. } \\
& \frac{p_{4}+1}{2}=\frac{89+1}{2}=45, \text { which is not a square. } \\
& \frac{p_{5}+1}{2}=\frac{97+1}{2}=49=7^{2} .
\end{aligned}
$$

A quick way to rule out 41, 73 and 89, as remarked by Ochem [12] over at Mathematics StackExchange, is as follows: "If $(p+1) / 2$ is an odd square, then $(p+1) / 2 \equiv 1(\bmod 8)$, so that $p \equiv 1$ (mod 16$)$. This rules out 41, 73, and 89."

So we are now in the following situation: Assuming $\sigma\left(m^{2}\right) / p^{k}$ is a square, we have $\sigma\left(m^{2}\right) \equiv 1$ $(\bmod 4)$ and $p \equiv k=1(\bmod 8)$.

Adjusting to account for $\sigma\left(m^{2}\right)$ modulo 8 and for $p, k$ modulo 16, we obtain either

$$
\sigma\left(m^{2}\right) \equiv 1 \quad(\bmod 8) \text { and } p \equiv 1 \quad(\bmod 16)
$$


or

$$
\sigma\left(m^{2}\right) \equiv 5 \quad(\bmod 8) \text { and } p \equiv 9 \quad(\bmod 16),
$$

from Remark 3.11. Furthermore, we know by Remark 4.1 that if $\sigma\left(m^{2}\right) / p^{k}$ is a square, then $p \equiv 1(\bmod 16)$.

This implies that the lowest possible value for the special prime $p$ is 17 .

We state this result as our next theorem.

Theorem 4.2. Suppose that $n=p^{k} m^{2}$ is an odd perfect number with special prime $p$. If $\sigma\left(m^{2}\right) / p^{k}$ is a square, then $\sigma\left(m^{2}\right) \equiv 1(\bmod 8)$ and $p \equiv 1(\bmod 16)$. It follows that $p \geq 17$.

\section{Evolution of the proof of a conjecture}

Additional tools are required if we are to push the lower bound for $p$ (when $\sigma\left(m^{2}\right) / p^{k}$ is a square) from 17 onwards.

On the other hand, we also know that the equation

$$
\operatorname{gcd}\left(m^{2}, \sigma\left(m^{2}\right)\right)=\frac{\sigma\left(m^{2}\right)}{p^{k}}=\frac{2 m^{2}}{\sigma\left(p^{k}\right)}=\frac{D\left(m^{2}\right)}{s\left(p^{k}\right)}=\frac{2 s\left(m^{2}\right)}{D\left(p^{k}\right)}
$$

holds, which is actually an identity.

Also, we have

$$
\operatorname{gcd}\left(m^{2}, \sigma\left(m^{2}\right)\right)=\frac{\sigma\left(m^{2}\right)}{p^{k}}=\frac{D\left(m^{2}\right)}{s\left(p^{k}\right)}=\frac{(p-1) D\left(m^{2}\right)}{p^{k}-1}
$$

from which we obtain

$$
\operatorname{gcd}\left(m^{2}, \sigma\left(m^{2}\right)\right)=\frac{\sigma\left(m^{2}\right)-(p-1) D\left(m^{2}\right)}{p^{k}-\left(p^{k}-1\right)}=2(1-p) m^{2}+p \sigma\left(m^{2}\right)
$$

and this last equation holds unconditionally. Finally, when $\sigma\left(m^{2}\right) / p^{k}$ is a square, then as discussed in [1] (Lemma 8, page 7), it follows that $k=1$, so that the odd perfect number $n=p^{k} m^{2}$ can be written in the form

$$
n=\frac{p(p+1)}{2} \cdot D\left(m^{2}\right)
$$

where both $(p+1) / 2$ and $D\left(m^{2}\right)$ are squares. Since $k=1$, then we consider whether it is possible that

so that, solving for $p$, we obtain

$$
2 m^{2}-\sigma\left(m^{2}\right)=D\left(m^{2}\right)=\frac{p+1}{2}
$$

$$
p=4 m^{2}-2 \sigma\left(m^{2}\right)-1 .
$$

Substituting this value in the other equation containing $p$ :

$$
\begin{aligned}
& 2 m^{2}-\sigma\left(m^{2}\right)=p \sigma\left(m^{2}\right)-2(p-1) m^{2} \\
& 2 m^{2}-\sigma\left(m^{2}\right)=\left(4 m^{2}-2 \sigma\left(m^{2}\right)-1\right) \sigma\left(m^{2}\right)-2\left(4 m^{2}-2 \sigma\left(m^{2}\right)-2\right) m^{2} \\
& 2 m^{2}-\sigma\left(m^{2}\right)=4 m^{2} \sigma\left(m^{2}\right)-2\left(\sigma\left(m^{2}\right)\right)^{2}-\sigma\left(m^{2}\right)-4\left(2 m^{2}-\sigma\left(m^{2}\right)-1\right) m^{2},
\end{aligned}
$$

which unfortunately, even after further simplification, does not lead to a contradiction. 
Nonetheless, we predict that:

Conjecture 5.1. Suppose that $n=p^{k} m^{2}$ is an odd perfect number with special prime $p$. If $\sigma\left(m^{2}\right) / p^{k}$ is a square, then

$$
D\left(m^{2}\right) \neq \frac{p+1}{2} .
$$

Remark 5.2. We end this section with some remarks about conditions which follow from assuming the negation of Conjecture 5.1.

First and foremost, we have

$$
\frac{\sigma\left(m^{2}\right)}{p}=D\left(m^{2}\right)=\frac{m^{2}}{(p+1) / 2}=\frac{p+1}{2},
$$

which implies that the odd perfect number $n=p^{k} m^{2}$ takes the form

$$
n=p\left(\frac{p+1}{2}\right)^{2}=(2 m-1) m^{2} .
$$

Notice that we then have the equations

$$
\sigma\left(\left(\frac{p+1}{2}\right)^{2}\right)=\sigma\left(m^{2}\right)=p D\left(m^{2}\right)=\frac{p(p+1)}{2}
$$

and inequalities

$$
\frac{p(p+1)}{2}=\sigma\left(\left(\frac{p+1}{2}\right)^{2}\right)<\left(\sigma\left(\frac{p+1}{2}\right)\right)^{2} \leq(p-1)^{2},
$$

where we have used the inequality $\sigma((p+1) / 2) \leq p-1$ (from the line immediately preceding the statement of Theorem 4 in page 5 of Cohen and Sorli's paper [4]).

This results in the trivial lower bound $p \geq 5$ - hence, still no contradiction, at this point.

Proof. As this article was about to be submitted to NNTDM, the authors realized how to prove Conjecture 5.1.

First, we need the following lemma, proved in https: / / math. stackexchange.com/ questions/3121498: If $n=p^{k} m^{2}$ is an odd perfect number with special prime $p$, then $m^{2}-p^{k}$ is not a square.

We reproduce the proof of the lemma in the following form here: If $n=p^{k} m^{2}$ is an odd perfect number with special prime $p$, then $m^{2}-p^{k}$ is not a square if $\sigma\left(m^{2}\right) / p^{k}$ is a square.

Let $p^{k} m^{2}$ be an odd perfect number with special prime $p$. Then $p \equiv k \equiv 1(\bmod 4)$ and $\operatorname{gcd}(p, m)=1$.

By Pomerance et al. [5], we know that $p^{k}<m^{2}$, so that $m^{2}-p^{k}$ is a positive integer. Also, since $m^{2}$ is a square and $p \equiv 1(\bmod 4)$, then

$$
m^{2}-p^{k} \equiv 1-1 \equiv 0 \quad(\bmod 4)
$$

Suppose that $p^{k} m^{2}$ is an odd perfect number with special prime $p$, and that $m^{2}-p^{k}=s^{2}$, for some integer $s \geq 2$. 
Then

$$
m^{2}-s^{2}=p^{k}=(m+s)(m-s)
$$

so that we obtain

$$
\left\{\begin{array}{l}
p^{k-v}=m+s \\
p^{v}=m-s
\end{array}\right.
$$

where $v$ is a positive integer satisfying $0 \leq v \leq(k-1) / 2$. It follows that we have the system

$$
\left\{\begin{array}{l}
p^{k-v}+p^{v}=p^{v}\left(p^{k-2 v}+1\right)=2 m \\
p^{k-v}-p^{v}=p^{v}\left(p^{k-2 v}-1\right)=2 s
\end{array}\right.
$$

Since $p$ is a prime satisfying $p \equiv 1(\bmod 4)$ and $\operatorname{gcd}(p, m)=1$, from the first equation it follows that $v=0$, so that we obtain

$$
\left\{\begin{array}{l}
p^{k}+1=2 m \\
p^{k}-1=2 s
\end{array}\right.
$$

which yields

$$
m=\frac{p^{k}+1}{2}<p^{k}
$$

Lastly, note that the inequality $p<m$ has been proved by Brown (2016) [2], Dris (2017) [7], and Starni (2018) [15], so that we are faced with the inequality

$$
p<m<p^{k} \text {. }
$$

This implies that $k>1$.

Now assume to the contrary that $D\left(m^{2}\right)=(p+1) / 2$ and $\sigma\left(m^{2}\right) / p^{k}$ is a square.

Then we obtain the following form for the odd perfect number $n=p^{k} m^{2}=p m^{2}$ :

$$
n=(2 m-1) m^{2} \text {. }
$$

It follows that

$$
m^{2}-p^{k}=m^{2}-p=m^{2}-(2 m-1)=m^{2}-2 m+1=(m-1)^{2}
$$

is a square. By our lemma, this implies that $k>1$.

This clearly contradicts $\sigma\left(m^{2}\right) / p^{k}$ being a square, since it implies $k=1$. QED

We end this section with a corollary to the proof of Conjecture 5.1:

Corollary 5.2.1. Suppose that $n=p^{k} m^{2}$ is an odd perfect number with special prime $p$. If $\sigma\left(m^{2}\right) / p^{k}$ is a square, then $D\left(m^{2}\right)>(p+1) / 2$.

Proof. The proof proceeds by contradiction and uses the inequality $p<m[2,7,15]$. 


\subsection{A simplified approach to Conjecture 5.1}

We include this subsection here for the sake of completion. (The authors realized these results after this paper was accepted by NNTDM, but prior to publication.)

First, we state and prove some quick lemmas.

Lemma 5.3. Suppose that $n=p^{k} m^{2}$ is an odd perfect number with special prime $p$. If the equation

$$
D\left(m^{2}\right)=\frac{p+1}{2}
$$

holds, then $k=1$.

Proof. Let $n=p^{k} m^{2}$ be an odd perfect number with special prime $p$. Suppose that the equation

$$
D\left(m^{2}\right)=\frac{p+1}{2}
$$

holds. Dividing through by $s\left(p^{k}\right)$, we get

$$
\frac{D\left(m^{2}\right)}{s\left(p^{k}\right)}=\frac{(p+1)(p-1)}{2\left(p^{k}-1\right)} .
$$

But we know that

$$
\frac{D\left(m^{2}\right)}{s\left(p^{k}\right)}=\frac{\sigma\left(m^{2}\right)}{p^{k}}
$$

and that $\sigma\left(m^{2}\right) / p^{k} \geq 3$ by Dris (2008) [6]. Therefore, we get the inequality

$$
3 \leq \frac{(p+1)(p-1)}{2\left(p^{k}-1\right)},
$$

which implies that

$$
6\left(p^{k}-1\right) \leq p^{2}-1
$$

Since the prime $p$ satisfies $p \equiv k \equiv 1(\bmod 4)$, then $p^{k} \geq 5$, which means that

$$
p^{k}-1<6\left(p^{k}-1\right) \leq p^{2}-1 \text {. }
$$

This gives $k<2$, from which we conclude that $k=1$ (since $k$ must be a positive integer satisfying $k \equiv 1(\bmod 4))$.

Lemma 5.4. Suppose that $n=p^{k} m^{2}$ is an odd perfect number with special prime $p$. If $k=1$, then the inequation

$$
D\left(m^{2}\right) \neq \frac{p+1}{2}
$$

holds.

Proof. Let $n=p^{k} m^{2}$ be an odd perfect number with special prime $p$. Suppose that $k=1$. Assume to the contrary that the equation

$$
D\left(m^{2}\right)=\frac{p+1}{2}
$$


holds. Dividing through by $m^{2}$, we get

$$
2-\frac{\sigma\left(m^{2}\right)}{m^{2}}=\frac{D\left(m^{2}\right)}{m^{2}}=\frac{p+1}{2 m^{2}}
$$

But since $k=1$ is true, then we have

$$
\frac{\sigma\left(m^{2}\right)}{m^{2}}=\frac{2 p^{k}}{\sigma\left(p^{k}\right)}=\frac{2 p}{p+1}
$$

Substituting, we obtain

$$
\frac{p+1}{2 m^{2}}=\frac{D\left(m^{2}\right)}{m^{2}}=2-\frac{\sigma\left(m^{2}\right)}{m^{2}}=2-\frac{2 p}{p+1}=\frac{2}{p+1},
$$

from which we finally get

$$
m^{2}=\left(\frac{p+1}{2}\right)^{2}
$$

This implies that

$$
m=\frac{p+1}{2}<p,
$$

which contradicts the result $p<m$ by Brown (2016) [2], Dris (2017) [7], and Starni (2018) [15].

Clearly, Lemma 5.3 and Lemma 5.4 together give a proof for the following theorem. (Note that this theorem proves and is stronger than Conjecture 5.1.)

Theorem 5.5. Suppose that $n=p^{k} m^{2}$ is an odd perfect number with special prime $p$. The inequation

$$
D\left(m^{2}\right) \neq \frac{p+1}{2}
$$

holds.

Proof. Let $n=p^{k} m^{2}$ be an odd perfect number with special prime $p$. Assume to the contrary that

$$
D\left(m^{2}\right)=\frac{p+1}{2}
$$

By Lemma 5.3, we have the implication

$$
D\left(m^{2}\right)=\frac{p+1}{2} \Longrightarrow k=1 \text {. }
$$

It follows that $k=1$. Furthermore, by Lemma 5.4, we get

$$
k=1 \Longrightarrow D\left(m^{2}\right) \neq \frac{p+1}{2} .
$$

This implies that

$$
D\left(m^{2}\right) \neq \frac{p+1}{2},
$$

which contradicts our initial assumption that

$$
D\left(m^{2}\right)=\frac{p+1}{2} .
$$

We therefore conclude that

$$
D\left(m^{2}\right) \neq \frac{p+1}{2} .
$$


The following theorem is a stronger version of Corollary 5.2.1.

Theorem 5.6. Suppose that $n=p^{k} m^{2}$ is an odd perfect number with special prime $p$. The inequality

$$
D\left(m^{2}\right)>\frac{p+1}{2}
$$

holds.

Proof. Let $n=p^{k} m^{2}$ be an odd perfect number with special prime $p$. Assume to the contrary that

$$
D\left(m^{2}\right) \leq \frac{p+1}{2}
$$

By Theorem 5.5, we have

$$
D\left(m^{2}\right) \neq \frac{p+1}{2}
$$

It follows that

$$
D\left(m^{2}\right)<\frac{p+1}{2} .
$$

Dividing through by $m^{2}$, we get

$$
2-\frac{\sigma\left(m^{2}\right)}{m^{2}}<\frac{p+1}{2 m^{2}} .
$$

But $\sigma\left(m^{2}\right) / m^{2}$ is bounded from above as follows:

$$
\frac{\sigma\left(m^{2}\right)}{m^{2}} \leq \frac{2 p}{p+1}
$$

This implies that

$$
\frac{2}{p+1}=2-\frac{2 p}{p+1} \leq 2-\frac{\sigma\left(m^{2}\right)}{m^{2}}<\frac{p+1}{2 m^{2}} .
$$

It follows that

$$
m^{2}<\left(\frac{p+1}{2}\right)^{2}
$$

from which we conclude that

$$
m<\frac{p+1}{2}<p,
$$

which contradicts the result $p<m$ by Brown (2016) [2], Dris (2017) [7], and Starni (2018) [15].

We therefore conclude that

$$
D\left(m^{2}\right)>\frac{p+1}{2} .
$$

\section{Further research}

It is currently not clear to the authors how to use Theorem 1.2 to further simplify and/or unify the presentation of the congruences in Remark 3.11 (where we have considered $\sigma\left(m^{2}\right)$ modulo 8), similar to those used by Chen and Luo in their theorem (where they considered $\sigma\left(m^{2}\right)$ modulo $4)$.

Likewise, the authors do not currently see a quick way to settle the following conjecture, which is a generalization of Theorem 5.5: 
Conjecture 6.1. Suppose that $n=p^{k} m^{2}$ is an odd perfect number with special prime $p$. The inequation

$$
\frac{D\left(m^{2}\right)}{s\left(p^{k}\right)} \neq \frac{\sigma\left(p^{k}\right)}{2}
$$

holds.

We leave these problems to other researchers for resolution.

\section{Acknowledgements}

The authors are indebted to the anonymous referees whose valuable feedback improved the quality of this manuscript.

\section{References}

[1] Broughan, K. A., Delbourgo, D., \& Zhou, Q. (2013). Improving the Chen and Chen result for odd perfect numbers, Integers, 13, Article \#A39.

[2] Brown, P. A. (2016). A partial proof of a conjecture of Dris, preprint, https: / / arxiv . org/abs/1602.01591.

[3] Chen, S.-C., \& Luo, H. (2013). Odd multiperfect numbers, Bull. Aust. Math. Soc., 88 (1), $56-63$.

[4] Cohen, G. L., \& Sorli, R. M. (2012). On odd perfect numbers and even 3-perfect numbers, Integers, 12A, Article \#A6.

[5] Dandapat, G. G., Hunsucker, J. L., \& Pomerance, C. (1975). Some new results on odd perfect numbers, Pacific J. Math., 57 (2), 359-364.

[6] Dris, J. A. B. (2008). Solving the odd perfect number problem: some old and new approaches, M. Sc. thesis, De La Salle University, Manila, Philippines.

[7] Dris, J. A. B. (2017). On a curious biconditional involving the divisors of odd perfect numbers, Notes Number Theory Discrete Math., 23 (4), 1-13.

[8] Dris, J. A. B. (2017). Conditions equivalent to the Descartes-Frenicle-Sorli conjecture on odd perfect numbers, Notes Number Theory Discrete Math., 23 (2), 12-20.

[9] Dris, J. A. B., \& San Diego, I. T. (2020). Some modular considerations regarding odd perfect numbers, Notes Number Theory Discrete Math., 26 (2), 27-33.

[10] Dris, J. A. B., \& MathStackExchange user FredH (2019). If $n=p^{k} m^{2}$ is an odd perfect number with special prime $p$, then $m^{2}-p^{k}$ is not a square. https://math. stackexchange.com/questions/3121498 
[11] Ewell, J. A. Jr. (1980). On the multiplicative structure of odd perfect numbers, J. Number Theory, 12, 339-342.

[12] Ochem, P. (2019). Answer to a question of the first author in Mathematics StackExchange, https://math.stackexchange.com/a/3151412/28816.

[13] Sloane, N. J. A., OEIS sequence A033879 - Deficiency of $n$, or $2 n-\sigma(n)$, https:// oeis.org/A033879.

[14] Sloane, N. J. A., \& Guy, R. K., OEIS sequence A001065 - Sum of proper divisors (or aliquot parts) of $n$ : sum of divisors of $n$ that are less than $n$, https: / / oeis.org/A001065.

[15] Starni, P. (2018). On Dris conjecture about odd perfect numbers, Notes Number Theory Discrete Math., 24 (1), 5-9.

[16] Starni, P. (1991). On the Euler's factor of an odd perfect number, J. Number Theory, 37, $366-369$.

[17] Wikipedia contributors. (2019, March 6). Perfect number. In Wikipedia, The Free Encyclopedia. Retrieved from https://en.wikipedia.org/w/index.php?title= Perfect_number\&oldid=934645565. 